\title{
La integración de la extensión, la docencia y la investigación universitarias: el caso del Proyecto Team-UNA de la Sede Regional Brunca
}

\section{The Integration of University Extension, Teaching and Research: The Case of Team-UNA Project at the Brunca Campus, Perez Zeledon, Costa Rica}

\author{
Lena Barrantes Elizondo \\ Universidad Nacional \\ Sede Regional Brunca, Campus Pérez Zeledón \\ Costa Rica, Pérez Zeledón \\ lenna.barrantes.elizondo@una.cr \\ Evelyn Valverde Marín \\ Universidad Nacional \\ Sede Regional Brunca, Campus Pérez Zeledón \\ Guanacaste, Costa Rica \\ evelyn.valverde.marin@una.cr \\ Emanuel Quesada Estrada \\ Universidad Nacional \\ Sede Regional Brunca, Campus Pérez Zeledón \\ Pérez Zeledón, Costa Rica \\ emma25QE@gmail.com
}

Recibido 04/12/2017 Aceptado 27/03/2018

Resumen. En la búsqueda de la calidad en la relación extensión-docencia-investigación se evidencia la necesidad de estar en constante replanteamiento de estrategias de integración. El Proyecto Team-UNA de la Sede Regional Brunca nació en el año 2010 con el propósito de trabajar con el sector estudiantil del Ciclo Diversificado de la Educación General Básica, para el mejoramiento de su calidad de vida a través de la oferta de tutorías en Inglés gratuitas, logrando así vincular la docencia y la extensión. Este proyecto busca gestionar la participación de estu- 
Revista Universidad en DiÁlogo • Vol. 8, N. ${ }^{\circ}$ 1, Enero-Junio, 2018, pp. 73-84

ISSN 2215-2849 • EISSN: 2215-4752

DOI: http://dx.doi.org/10.15359/udre.8-1.5

diantes de la carrera Bachillerato en la Enseñanza del Inglés como tutores y tutoras de estudiantes de secundaria procedentes de colegios públicos de la región.

Este estudio tiene como objetivo dar a conocer la experiencia generada, como base para fortalecer el proceso de enseñanza y aprendizaje vivencial. La sistematización se llevó a cabo a través de la recolección de experiencias de los actores principales por medio de su participación en grupos focales a lo largo del desarrollo del proyecto. Como resultado, se llega a la conclusión de que Team-UNA es un catalizador de aprendizaje vivencial que fomenta el análisis crítico, la toma de decisiones y la resolución de problemas, elementos necesarios que respaldan la integración de la docencia, la extensión y la investigación educativas.

Palabras clave: aprendizaje vivencial, estrategia de integración, extensión, docencia, investigación.

Abstract. When aiming at attaining a sound relationship between extension, teaching, and research, it is evident that a constant rethinking of integration strategies is mandatory. Team-UNA Project at Brunca Campus, Perez Zeledon, Costa Rica, started in 2010; its purpose was to work with 10th to 12th high school students in an attempt to improve their quality of life through offering English tutoring sessions at no cost. In this way, teaching and extension were partnered. The main objective of this project is to enable the participation of university students from the English Teaching Major as tutors of students from public high schools in the surrounding community. It is with this perspective that this article intends to disseminate the experiences generated by these university students as a basis for strengthening the experiential learning process. For this study, data systematization was made available through the collection of the experiences of the participants through their involvement in focus groups that took place during the development of the project. As a result, we conclude that Team-UNA Project is a catalyst for experiential learning that promotes critical analysis, decision making and problem resolution, which are key elements that underpin the sound integration of university teaching, extension, and research.

Keywords: experiential learning, integration strategies, extension, teaching, research.

\section{Introducción}

La misión de la universidad pública es convertirse en referencia del conocimiento en los diferentes campos del saber. La clave de esta misión está en poder establecer una estrecha relación entre la docencia, la extensión y la investigación. En la búsqueda de la calidad de este vínculo, se 
evidencia la necesidad de estar en constante replanteamiento de estrategias de integración. Un ejemplo es el Proyecto Team-UNA, el cual se desarrolla en las instalaciones de la Universidad Nacional, Sede Regional Brunca (UNASRB). Este proyecto se desarrolla como un proceso de dos vías, debido a que estudiantes de la carrera Bachillerato en la Enseñanza del Inglés (BEI) ofrecen tutorías de Inglés gratuitas a estudiantes de secundaria de la educación pública, específicamente del Ciclo Diversificado de la Educación General Básica. A la vez, al estar envuelta en el desarrollo del proyecto y al tener una activa participación en todas sus etapas, la comunidad estudiantil universitaria tiene la oportunidad de analizar en forma crítica todos los detalles del proceso, para así extraer enseñanzas y poder aplicar lo aprendido en su desempeño como futuros y futuras docentes. Además, la participación del estudiantado en las tutorías ya ha demostrado proveerles herramientas de aprendizaje concretas y ha desarrollado una visión humanista (Barrantes, 2016). Así, el objetivo de este estudio es dar a conocer la experiencia generada a través de este proyecto y, a través de su ejemplo, incorporar aún más el aprendizaje vivencial, para convertirlo en un elemento fundamental de la extensión universitaria.

\section{Generalidades del Proyecto Team-UNA}

Desde sus inicios, la educación se ha caracterizado por brindar a la sociedad conocimientos y herramientas que les permitan a sus ciudadanos y ciudadanas prepararse para enfrentar las vivencias que les ofrezca la vida, sin importar su credo, raza o nivel social. Sin embargo, la educación va más allá de la experiencia en el aula. Las universidades públicas y, en particular, la Universidad Nacional de Costa Rica (UNA) se han comprometido con las necesidades que la sociedad presenta. El Estatuto Orgánico de la UNA (2015) estableció que:

La acción sustantiva de la universidad se realiza mediante la docencia, la investigación, la extensión, la producción, y otras formas que establezca la normativa institucional, las cuales se complementan y nutren mutuamente. Integra diversas prácticas y propicia el diálogo entre saberes, de manera innovadora, sistemática y transformadora. Responde a los principios, valores y fines estatutarios, a las necesidades de desarrollo de la sociedad, a sus políticas públicas, al desarrollo científico y tecnológico y a la formación integral de las personas. (p. 23) 
Revista Universidad en DiÁlogo • Vol. 8, N. ${ }^{\circ}$ 1, Enero-Junio, 2018, pp. 73-84

ISSN 2215-2849 • EISSN: 2215-4752

DOI: http://dx.doi.org/10.15359/udre.8-1.5

Por el compromiso y responsabilidad social que la universidad ha adquirido, han surgido múltiples proyectos con un nivel de interdisciplinariedad. Tanto docentes como estudiantes forman parte de este quehacer. Los y las docentes, con sus conocimientos, desarrollan el proyecto; mientras que la población estudiantil pone en práctica sus destrezas en un contexto real y desarrolla aquellas que solo se pueden favorecer empíricamente.

Un ejemplo de la acción sustantiva que la UNASRB ejerce en las comunidades es el Proyecto Team-UNA. Este es un proyecto dirigido a la comunidad que se desarrolla en las instalaciones del Campus Pérez Zeledón. Desde el 2010, estudiantes de la Universidad Nacional dan tutorías de Inglés gratuitas a estudiantes de secundaria de la educación pública. En la justificación del proyecto se encuentra la importancia de ayudar a la comunidad y de formar a la población estudiantil universitaria más allá del área lingüística. A través de Team-UNA, la Sede Brunca busca "a formación de profesionales de excelencia con conciencia social y humanística que sean capaces de identificar las necesidades del desarrollo regional" (Barrantes, 2016, p. 96) en su área de conocimiento. Esta actividad les da la oportunidad a los y las estudiantes de la carrera BEI de formarse como profesores y profesoras, ya que provee un espacio en donde la teoría y la práctica se confrontan. Este espacio les permite realizar actividades de selección de escenarios, diagnóstico, observación, diseño del planeamiento didáctico y aplicación de una variedad de metodologías aprendidas en el aula.

El aporte del proyecto al fortalecimiento social consiste en ser una actividad de aprendizaje de servicio donde los componentes de extensión y docencia se integran. El fin es brindar a la comunidad estudiantil de secundaria un segundo espacio de aprendizaje del idioma inglés, en el cual se enfatice la comprensión de lectura. El énfasis que se le da a la enseñanza del inglés tiene como principio proveer a los y las estudiantes de secundaria herramientas que les permitan alcanzar el éxito académico en el año escolar que cursan, obtener altas calificaciones en su prueba de bachillerato y dotarse de capacidades lingüísticas que les facilitarán su inmersión en la vida universitaria.

\section{Estructuración metodológica del proyecto}

El enfoque comunicativo, las técnicas de lectura, el aprendizaje cooperativo, las tutorías, el trabajo en equipo y el liderazgo son los pilares metodológicos 
para la ejecución del proyecto. Estos principios teóricos desarrollan criterios de conducta en las personas tutoras que les ayudan a formarse como profesionales altamente equipadas, no solo con estrategias de enseñanza, sino con herramientas sociales que les permitirán formar parte de manera efectiva de un grupo social establecido para alcanzar metas a corto y largo plazo. Cada tutor o tutora asumirá su papel de líder y responsable de la ejecución exitosa de cada sesión de trabajo. Las opiniones y decisiones de los tutores y las tutoras son tomadas en cuenta de manera significativa, ya que serán quienes a través de la práctica profesional y su análisis crítico de las circunstancias identifiquen debilidades y virtudes del proyecto.

El enfoque comunicativo es la base metodológica para las tutorías. Se implementan actividades interactivas para el aprendizaje de vocabulario, se refuerza la pronunciación y se provee al o a la estudiante con estrategias para la comprensión de lectura. Se da énfasis a la habilidad de lectura y microhabilidades de vocabulario y gramática. Las estrategias de lectura que se enfatizan son skimming, scanning, lectura activa y adivinanza contextual (Larsen-Freeman, 2011).

\section{Participación estudiantil}

Una particularidad de este proyecto es la activa participación de los y las estudiantes en todas sus etapas. Las actividades se dividen en tres fases: logística, desarrollo y sistematización final. En la primera, las personas que brindan tutorías realizan un proceso de captación de otras personas tutoras y de estudiantes participantes, para lo que realizan visitas a los diferentes colegios. Son quienes comparten la información con la población estudiantil y establecen mecanismos para su matrícula. Además, analizan el material por utilizar, diseñan instrumentos de evaluación y sugieren actividades de mediación.

Durante la segunda etapa, se encargan de impartir las tutorías y de resolver todas las situaciones que comprometan la ejecución de cada sesión. Las tutoras y los tutores también participan de reuniones mensuales en donde se discuten lineamientos y posibles eventualidades del proyecto. Estas reuniones son un espacio para socializar experiencias, reflexionar sobre ellas y tomar decisiones sobre posibles cambios o prácticas de fortalecimiento. También participan de talleres pedagógicos en donde se refuerzan estrategias de enseñanza y habilidades de liderazgo. 
Revista Universidad en DiÁlogo • Vol. 8, N. ${ }^{\circ}$ 1, Enero-Junio, 2018, pp. 73-84

ISSN 2215-2849 • EISSN: 2215-4752

DOI: http://dx.doi.org/10.15359/udre.8-1.5

En la tercera etapa, las tutoras y los tutores comparten sus experiencias para la mejora continua del proyecto mediante su participación en grupos focales o discusiones de grupo. Finalmente, con la guía de las coordinadoras, proveen insumos para la redacción de un informe anual.

En la carrera BEI, en la UNASRB, se cuenta con estudiantes completamente comprometidos y comprometidas con su comunidad, con alta capacidad de liderazgo y cooperación activa. Estos elementos los y las ayudan a crecer y alcanzar el éxito como docentes competentes que desean involucrarse en las necesidades de su comunidad. Desde el punto de vista de LarsenFreeman (2011), el aprendizaje cooperativo implica esencialmente que el estudiantado aprenda en grupos con sus compañeras y compañeros. Pero no es la configuración del grupo lo que hace que el aprendizaje cooperativo sea distintivo, es la forma en la que trabajan juntos el estudiantado y el cuerpo docente. Este supuesto es un ejemplo de cómo la contribución entre docentes y estudiantes facilita el desarrollo del lenguaje y el crecimiento profesional. Durante el proceso de aprendizaje de un segundo idioma, tienen la oportunidad de comprender que su objetivo requiere más que solo asistir todos los días a clases. Poco pueden alcanzar si pasan por alto la realidad que se vive dentro de una institución educativa. El Team-UNA permite que la población estudiantil tenga una participación activa en la construcción de sus propias experiencias, al ofrecerle la oportunidad de vivenciar retos en contextos reales.

Para el proceso de selección de tutores y tutoras se invita a cualquier estudiante del BEI que esté interesado o interesada en fortalecer sus habilidades pedagógicas y su liderazgo, así como en poseer rasgos de conciencia social y humanística que le den la capacidad de identificar las necesidades de su comunidad. El departamento de Dirección Académica nombra estudiantes asistentes por recomendación de las coordinadoras del proyecto. Regularmente, estas personas son estudiantes que han dado tutorías anteriormente y que demuestran compromiso y afinidad. Existen dos tipos: tutor o tutora responsable y tutor o tutora asistente. El primer tipo lidera las sesiones durante las primeras cuatro semanas y guía a la persona tutora asistente en el proceso. El segundo tipo asiste a la persona tutora responsable con algunas tareas como revisar prácticas, facilitar material y aclarar consultas.

La manera en que se concretiza la participación de las y los estudiantes en este proyecto permite que experimenten de manera efectiva las cuatro etapas del aprendizaje vivencial: experiencia concreta, observación reflexiva, 
conceptualización abstracta y la experimentación activa. Es así como la comunidad estudiantil universitaria construye su propio conocimiento, adquiere habilidades y realza sus valores directamente desde la experiencia, al ser parte activa y fundamental de las tutorías.

\section{Recolección de experiencias}

Para esta recolección se contó con la participación de personas tutoras que fueron y son parte del grupo de Team-UNA durante los años 2010-2017. Para esto, se les realizó una entrevista y participaron en un grupo focal donde tuvieron la oportunidad de compartir tanto sus vivencias como el aporte del proyecto en su preparación profesional. En la fase de análisis de las experiencias se llevaron a cabo muchos aprendizajes, que ayudaron a confirmar la importancia del proyecto como un espacio que propicia la toma de decisiones, el posicionamiento del Team-UNA como grupo de apoyo, la oportunidad de las personas participantes de desarrollar habilidades pedagógicas afines a su área, el fortalecimiento de la confianza de los y las participantes y el convencimiento de las personas tutoras por extender la participación estudiantil.

A través de la información recolectada, se evidenció que el proyecto tiene un rol significativo, al guiar a las personas tutoras y potenciar sus capacidades y conocimientos de manera práctica. Estas han sido visibles cuando llegan a ejercer como docentes en alguna institución educativa, ya que han tenido contacto real con ambientes de enseñanza donde es forzoso tomar decisiones asertivas en la aplicación de las metodologías o técnicas. La toma de decisiones fue un tema fundamental recalcado por las personas entrevistadas. La vincularon con el trabajo en equipo, ya que la unión de las personas tutoras en diferentes periodos y actividades les ayudó a sentir seguridad para decidir qué hacer en momentos de crisis.

Una de las tutoras comentó: "Esas sesiones para resolver problemas o tomar decisiones fue lo que me hicieron capaz de ahora poder hacerlo sola y sentir la confianza de que lo estoy haciendo bien" (Comunicación personal, 2017). Las personas tutoras también compartieron experiencias positivas con respecto al desarrollo de otras capacidades fundamentales, como demostrar una mente más crítica, un buen manejo del grupo, precisión para dar instrucciones, técnicas claras para la explicación de temas complicados y el desarrollo de una creatividad sustancial. Dichas habilidades son transcendentales para lograr tutorías exitosas.- 
Revista Universidad en DiÁlogo • Vol. 8, N. ${ }^{\circ}$ 1, Enero-Junio, 2018, pp. 73-84

ISSN 2215-2849 • EISSN: 2215-4752

DOI: http://dx.doi.org/10.15359/udre.8-1.5

El acompañamiento en la formación docente es una estrategia de aprendizaje que el proyecto ha acuñado desde su concepción. En cuanto a este tema, Huling-Austin (1992) considera los grupos de apoyo como un importante complemento a la ayuda individualizada, señalando entre sus ventajas el hacer menos egocéntrico al personal docente, reducir sus problemas de ansiedad y favorecer un mejor ajuste en las tareas de la enseñanza. TeamUNA no pretende adoptar un rol de mentorazgo con vínculo jerárquico, que establece a la persona mentora como poseedora de conocimiento experto; por el contrario, se anhela poder ser un grupo de apoyo en donde los y las estudiantes puedan "compartir experiencias, materiales, problemas, estrategias de actuación, comportamientos y actitudes en contextos libres de la amenaza de la evaluación de los compañeros más experimentados o los superiores" (Eirín y Montero, 2009 p. 103).

Según las personas entrevistadas, mediante sus experiencias como tutoras, dicho proyecto les brindó la oportunidad de vitalizar la responsabilidad, la tolerancia, la puntualidad, la disciplina, la constancia y el liderazgo. Los valores mencionados son el pilar para mostrar una interacción más fluida y exitosa. Por lo tanto, este aprendizaje les ayudó no solo a propiciar experiencias favorables, sino que también les permitió experimentar de primera mano y reflexionar acerca de la importancia y las consecuencias directas de adoptar estos valores y actitudes.

Uno de los temas más recalcados fue el de las experiencias en el desarrollo profesional como docentes de un idioma extranjero. El proyecto les provee un contacto directo con las metodologías pedagógicas sugeridas, así como los aspectos didácticos y evaluativos, ya que estos son discutidos, analizados y aplicados en el proceso de planeamiento de las tutorías. Durante las reuniones mensuales, las tutoras y los tutores tienen la oportunidad de hacer cambios que consideren necesarios, ya sea en el material, las actividades, las evaluaciones o cualquier aspecto que, con base en argumentos válidos, consideren favorable para el desarrollo de las tutorías. Este espacio de discusión les permite generar ideas críticas y establecer estrategias de comunicación efectivas. Para sugerir un cambio, las personas tutoras deben demostrar conocimiento del tema y ser capaces de convencer a sus compañeros y compañeras de los posibles beneficios de esos cambios. En muchas ocasiones, el ciclo de discusión, reflexión y aplicación se ha tenido que repetir cuando la estrategia sugerida no ha funcionado, lo que fortalece aún más el proceso de aprendizaje. 
Otro de los temas que surgieron fue el fortalecimiento de la confianza. Ser parte del proyecto les permitió a las tutoras y los tutores mejorar su autoconfianza, ya que reconocieron sus capacidades docentes en situaciones de enseñanza reales. Comentaron que el verse capaces de lidiar con situaciones que ponían a prueba sus capacidades les dio la seguridad para reducir sus problemas de ansiedad y falta de confianza. Muchas de las personas tutoras reconocieron, además, que se sienten capaces de controlar "el miedo escénico" frente a sus estudiantes de educación media. Aunado a esto, identificaron cómo pudieron transferir esta capacidad a sus clases universitarias, al aumentar la confianza durante su participación en actividades que requieren hablar en público, como presentaciones orales y mesas redondas. La inseguridad que produce la falta de conocimiento y la incertidumbre de no saber qué hacer obstaculiza la capacidad de comunicación y de mediación pedagógica del personal docente novato. Las experiencias de las tutoras y los tutores evidencian cómo esta inseguridad se ve transformada a través de su participación en las actividades del proyecto.

Una de las preguntas clave de la entrevista confrontó a las personas participantes sobre si al volver al pasado decidirían formar parte de Team-UNA, y todas afirmaron estar dispuestas a participar en el proyecto; de hecho, aseveraron que lo han recomendado a estudiantes de la carrera, pues opinan que "se obtienen muchos beneficios como experiencia, conocimiento de estrategias de estudio, aprendizaje, manejo de grupo, interacción, entre otras" (Comunicación personal, 2017). El recomendar a estudiantes formar parte del proyecto prueba el efecto significativo que tiene en la población estudiantil universitaria.

\section{El aprendizaje vivencial en el proceso de extensión universitaria}

Con base en la premisa de que se puede enseñar cada vez mejor, consideramos el aprendizaje vivencial no solo como una oportunidad de aprendizaje alternativo a la clase tradicional, sino también como una necesidad de nuestro tiempo en la lucha por proveer al estudiantado universitario con herramientas de aprendizaje acordes con la actualidad. La idea de ir más allá del aula nos impulsa a integrar en la estructura curricular espacios concretos de este tipo de aprendizaje y verlo como un componente de la enseñanza.

El aprendizaje vivencial optimiza las posibilidades del estudiantado de experimentar/vivenciar/enfrentar por sí mismo los retos de su profesión antes de graduarse. Esto les permite no solo familiarizarse con sus futuras 
Revista Universidad en DiÁlogo • Vol. 8, N. ${ }^{\circ}$ 1, Enero-Junio, 2018, pp. 73-84

ISSN 2215-2849 • EISSN: 2215-4752

DOI: http://dx.doi.org/10.15359/udre.8-1.5

responsabilidades, sino que también es un tiempo de reflexión sobre su afinidad con la profesión que han escogido. Según Kolb (1984), quien es el responsable de esta teoría, para que haya un aprendizaje efectivo el o la estudiante debería pasar por un proceso que incluye cuatro etapas. Este proceso se ha representado por medio de un modelo llamado ciclo del aprendizaje o ciclo de Kolb. Las cuatro etapas del ciclo son: experiencia concreta, observación reflexiva, luego de esta reflexión el o la estudiante es capaz de obtener conclusiones o generalizaciones que llevan a la conceptualización abstracta y, finalmente, a la experimentación activa, en donde prueba en la práctica las conclusiones obtenidas.

Según Jarvis (2007), el aprendizaje vivencial nace de la premisa de que el aprendizaje no está arraigado al presente, sino que por el contrario está orientado al futuro. Esto porque se entiende que la raíz del conocimiento y de la acción se encuentran en el corazón de la respuesta del ser humano hacia su mundo cotidiano y no en una simple acción instintiva que lo hace actuar de manera repetitiva. El aprendizaje es una necesidad social que lleva al ser humano a saber ser y a saber responder a sus demandas. El aprendizaje vivencial se puede visualizar como "aprender andando o haciendo", ya que es a través de la inmersión en situaciones reales que el estudiantado descubre sus propias respuestas o soluciones a conflictos presentes que podrán aplicar en el futuro. Este proceso requiere elaboración y acompañamiento. A pesar de que a la población estudiantil no se le dice qué hacer, qué sentir o cómo actuar, existe la guía de parte de los y las docentes, quienes proveen espacios de socialización, discusión y capacitación. No se debe confundir el aprendizaje vivencial con la experimentación. Un elemento clave de cómo el Team-UNA ha establecido esta diferencia es por medio de un proceso en donde los y las estudiantes que brindan tutorías socializan sus experiencias, reflexionan sobre ellas y las vuelven a enfrentar. En su papel de tutores y tutoras están enfrentando continuamente retos que comparten con su coordinadora y con sus pares. Esto les permite incorporar sus experiencias en su aprendizaje.

\section{Conclusiones}

En resumen, el Team-UNA es un catalizador de aprendizaje vivencial que fomenta el análisis crítico, la toma de decisiones y la resolución de problemas, los cuales son elementos necesarios que respaldan la integración de la docencia e investigación educativas. Team-UNA actualmente es un 
proyecto de extensión separado, pero dirigido al BEI. Se ha desarrollado de manera complementaria con la docencia. Sus actividades se han articulado bajo su propia agenda, sin ninguna vinculación directa con la estructura curricular de la carrera. Esto ha generado la inquietud por proponer una segunda etapa del proyecto, la cual apunte hacia una mejor integración. Hemos comprobado que existen espacios en la estructura curricular de los cursos pedagógicos en donde el aprendizaje vivencial y la investigación pueden ser vinculados en actividades propias del Team-UNA.

Finalmente, como parte de la participación en el III Congreso de Extensión Universitaria "Universidad Nacional: Dialogando en Territorios. Concepto y Políticas de Extensión 2018-2028", se generan las siguientes recomendaciones y aportes para la construcción de la política de extensión:

- Exponer mediante la sistematización de un proyecto de extensión su vinculación con las otras áreas (docencia e investigación) de la acción sustantiva de la Universidad

- Establecer la extensión universitaria como espacio de aprendizaje vivencial

- Reconocer la capacidad de la Sede Brunca para fortalecer el concepto de universidad democrática y comprometida socialmente

- Reconocer el proceso interactivo entre el conocimiento y su medio

- Reforzar la función social de la universidad orientada a la atención de problemáticas vinculadas con la educación

\section{Referencias}

Barrantes, L. (Enero-junio, 2016). Universitarios con conciencia social y humanista: el caso Team-UNA. Universidad en Diálogo, 6(1), 95-109.

Eirín, R. y Montero, L. (2009). Profesores principiantes e iniciación profesional. Estudio exploratorio. Profesorado. Revista de Currículum y Formación del Profesorado, 13(1), 101-115.

Huling-Austin, L. (1992). Research on learning to teach: Implications for teacher education and mentoring programs. Journal of Teacher Education, 3(43), 173-180. 
Revista Universidad en DiÁlogo • Vol. 8, N. ${ }^{\circ}$ 1, Enero-Junio, 2018, pp. 73-84

ISSN 2215-2849 • EISSN: 2215-4752

DOI: http://dx.doi.org/10.15359/udre.8-1.5

Jarvis, P. (2007). Globalisation, lifelong learning and the learning society: Sociological Perspectives. London: Routledge.

Kolb, D. (1984). Experiential learning: Experience as the source of learning and development. Englewood Cliffs, NJ: Prentice-Hall.

Larsen-Freeman, D. (2011). Techniques and principles in language teaching. New York: Oxford University Press.

UniversidadNacional.(2015).Estatuto Orgánico. Recuperadodehttp://www. documentos.una.ac.cr/bitstream/handle/unadocs/6693/ESTATUTOORGÁNICO-UNA-digital.pdf?sequence=1\&isAllowed=y 